\title{
A Proof of the Jordan Curve Theorem
}

\author{
Xing Zhang \\ School of Mathematics and Statistics, Wuhan University, Wuhan, China \\ Correspondence to: Xing Zhang, WHUZhangxing@gmail.com \\ Keywords: Jordan Curve Theorem, JCT, Tverberg, Jordan Polygon, Sausage Domain, Jordan Curve \\ Received: October 29, $2019 \quad$ Accepted: December 17, $2019 \quad$ Published: December 20, 2019 \\ Copyright $\odot 2019$ by author(s) and Scientific Research Publishing Inc. \\ This work is licensed under the Creative Commons Attribution International License (CC BY 4.0). \\ http://creativecommons.org/licenses/by/4.0/ \\ (c) (i) Open Access
}

\section{ABSTRACT}

The proof of the Jordan Curve Theorem (JCT) in this paper is focused on a graphic illustration and analysis ways so as to make the topological proof more understandable, and is based on the Tverberg's method, which is acknowledged as being quite esoteric with no graphic explanations. The preliminary constructs a parametrisation model for Jordan Polygons. It takes quite a length to introduce four lemmas since the proof by Jordan Polygon is the approach we want to concern about. Lemmas show that JCT holds for Jordan polygon and Jordan curve could be approximated uniformly by a sequence of Jordan polygons. Also, lemmas provide a certain metric description of Jordan polygons to help evaluate the limit. The final part is the proof of the theorem on the premise of introduced preliminary and lemmas.

\section{INTRODUCTION}

Though the definition of the Jordan Curve Theorem is not hermetic at all, the proof of the theorem is quite formidable and has experienced ups and downs throughout history.

Bernard Bolzano was the first person who formulated a precise conjecture: it was not self-evident but required a hard proof. However, the Jordan Curve Theorem was named after Camille Jordan, a mathematician who came up with the first proof in his lectures on real analysis and published his findings in his book [1], yet critics doubted the completeness of his proof. After that, using very complicated methods in 1905, Oswald Veblen was generally recognized as the first person who rigorously proved the Jordan Curve Theorem [2]. Veblen also commented that “His (Jordon's) proof, however, is unsatisfactory to many mathematicians. It assumes the theorem without proof in the important special case of a simple polygon, and of the argument from that point on, one must admit at least that all details are not given" [2]. However, in 2007, Thomas C. Hales demurred Veblen's comments and wrote that "Nearly every modern citation that I have found agrees that the first correct proof is due to Veblen ... In view of the heavy criticism of Jordan's proof, I was surprised when I sat down to read his proof to find nothing objectionable about it. Since then, I have contacted a number of the authors who have criticized Jordan, and each case the author has admitted to having no direct knowledge of an error in Jordan's proof" [3]. Hales also 
explained that the special case of simple polygons is not an easy exercise, but is not really in the way of Jordan's proof. He quoted Michael Reeken as saying “Jordan's proof is essentially correct ... Jordan's proof does not present the details in a satisfactory way. But the idea is right, and with some polishing, the proof would be impeccable" [3]. Just like Hales's judgement that vindicates the significance of Jordan's work, Schoenflies critically analyzed and completed Jordan's proof in 1924 [4]. However, the settlement of most controversies brought by Jordan's prove didn't diminish the enthusiasm of proving the Jordan Curve Theorem. Elementary proofs were presented by Filippov [5] in 1950 and Tverberg [6] in 1980. Maehara in 1984 used the Brouwer fixed point theorem to prove it [7]. A proof using non-planarity of the complete bipartite graph K3,3 was given by Thomassen in 1992 [8]. Although the Jordan Curve Theorem had been successfully proved in the 20th century, mathematicians still sought more formal ways to prove it in the 21 st century. The formal proof was provided by an international team of mathematicians using the Mizar system in 2005 and Hales in 2007 [3], respectively, and both of which rely on libraries of previously proved theorems.

This paper is intended to strengthen Tverberg's claim. Tverberg once suggested in his paper that "Although the JCT is one of the best known topological theorems, there are many, even among professional mathematicians, who have never read a proof of it. The present paper is intended to provide a reasonably short and self-contained proof or at least, failing that, to point at the need for one" [6]. Tverberg's paper is hard to understand generally reflected by those who have read it. So the following parts will support more details and graphs, so as to make the way of the proof more scrutable and credible.

It is comparatively easy to prove that the Jordan curve theorem holds for every Jordan polygon in Lemma 1, and every Jordan curve can be approximated arbitrarily well by a Jordan polygon in Lemma 2. Then Lemma 3 and Lemma 4 deal with the situation in limiting processes to prevent the cases from the polygons that may thin to zero somewhere.

\section{PRELIMINARIES}

Let $C$ be the unit circle $\left\{(x, y) \mid x^{2}+y^{2}=1\right\}$, a Jordan curve $\Gamma$ is the image of $C$ under an injective continuous mapping $\gamma$ into $\mathbb{R}^{2}$, i.e., a simple closed curve on the plane. We have the following fundamental fact.

Jordan Curve Theorem [1] (JCT): $\mathbb{R}^{2} \backslash \Gamma$ has exactly two connected components.

We introduce here some terms from analysis that will be used later.

First, we may recall some basic knowledge in real analysis.

We say that $M \in \mathbb{R}$ is a lower bound for a set $S \subset \mathbb{R}$ if each $s \in S$ satisfies $s \geq M$. And a lower bound for $S$ that is greater than all other lower bounds for $S$ is a greatest lower bound for $S$. The greatest lower bound for $S$ is denoted $g . l . b(S)$ or $\inf (S)$.

Since $C$ is compact, the mapping $\gamma$ is uniformly continuous on $C$, its inverse $\gamma^{-1}: \Gamma \rightarrow C$ is also uniformly continuous. Next, if $A$ and $B$ are nonempty disjoint compact sets in $\mathbb{R}^{2}$, then

$$
d(A, B):=\inf \{|a-b|: a \in A, b \in B\}
$$

Note that $d(A, B)$ is always positive, since, otherwise we have a sequence of points $\left(a_{n}\right),\left(b_{n}\right)$, $n \in \mathbb{N}$, so that $\left|a_{i}-b_{i}\right| \rightarrow 0$ as $i \rightarrow \infty$. Since $A$ is compact, $\left(a_{n}\right)$ has a subsequential limit, say $a \in A$, so that for any $\epsilon$ and sufficiently large $N$, by triangular inequality,

$$
\left|a-b_{N}\right| \leq\left|a-a_{N}\right|+\left|a_{N}-b_{N}\right| \leq \epsilon
$$

then $a$ is a limit point of $B$ thus $a \in B$ contradicts to $A \cap B=\varnothing$.

We assume that the unit circle $C$ is consistent with the natural parametrisation $t(\theta)=(\cos \theta, \sin \theta), \theta \in[0,2 \pi]$. A Jordan curve $\Gamma$ is said to be a Jordan polygon if there is a partition $\Theta=\left\{\theta_{0}, \theta_{1}, \cdots, \theta_{n}\right\}$ of the interval $[0,2 \pi]$ (i.e., $\left.0=\theta_{0}<\theta_{1}<\cdots<\theta_{n}=2 \pi\right)$, such that

$$
\gamma((\cos \theta, \sin \theta))=\left(a_{i} \theta+b_{i}, c_{i} \theta+d_{i}\right)
$$


for $\theta \in\left[\theta_{i-1}, \theta_{i}\right], \quad i \in\{1, \cdots, n\}, a_{i}, b_{i}, c_{i}, d_{i}$ are all real constants.

We call the pair $(\gamma, \Theta)$ a realisation of a polygon $\Gamma$.

Remark. In accordance with the choice of partition $\Theta$, we could define edges and vertices in a natural way. It is possible that adjacent edges of a polygon $\Gamma$ lie on the same line (Figure 1 ).

\section{LEMMAS}

We divide the proof of JCT into several steps. Lemma 1 below shows that JCT indeed holds for Jordan polygons. Lemma 2 shows every Jordan curve could be approximated uniformly by a sequence of Jordan polygons. Lemmas 3 and 4 provide certain metric description of Jordan polygons, which helps to evaluate the limit.

Lemma 1. The Jordan curve theorem holds for every Jordan polygon $\Gamma$ with realisation $(\gamma, \Theta)$.

Proof. Denote edges of $\Gamma$ to be $E_{1}, E_{2}, \cdots, E_{n}$, and vertices to be $v_{1}, v_{2}, \cdots, v_{n}$, (so $E_{i}=\gamma\left(\left(\theta_{i-1}, \theta_{i}\right)\right)$ and $\left.v_{i}=\gamma\left(\theta_{i}\right)\right)$ with

$$
E_{i} \cap E_{i+1}=\left\{v_{i}\right\}\left(i=1, \cdots, n, E_{n+1}=E_{1}, v_{i+1}=v_{1}\right)
$$

1) $\mathbb{R}^{2} \backslash \Gamma$ has at most two components.

Let $\delta:=\min \left\{d\left(E_{i}, E_{j}\right) \mid E_{i}\right.$ and $E_{j}$ are not adjacent $\}$ and let $N_{i}:=\left\{q \in \mathbb{R}^{2} \mid d\left(q, E_{i}\right)<\delta\right\}$ (Figure 2).

By definition no points of $N_{i}$ belongs to $E_{j}$, where $j \neq i-1, i, i+1$ (recall that $E_{0}=E_{n}$ ). so $N_{i} \cap \Gamma \subset E_{i-1} \cup E_{i} \cup E_{i+1}$ and it is clear that $N_{i} \backslash \Gamma$ consists of two connected components, say, $N_{i}^{\prime}$ and $N_{i}^{\prime \prime}$. We may assume, by elementary analytic geometry, (Figure 3 )

$$
N_{i}^{\prime} \cap N_{i+1}^{\prime} \neq \varnothing, N_{i}^{\prime \prime} \cap N_{i+1}^{\prime \prime} \neq \varnothing, i=1, \cdots, n
$$

Therefore $N_{1}^{\prime} \bigcup \cdots \cup N_{n}^{\prime}$ and $N_{1}^{\prime \prime} \bigcup \cdots \cup N_{n}^{\prime \prime}$ are both connected. For any $p$ in $\mathbb{R}^{2} \backslash N_{i}$, we could find a line segment from $p$ with length between $d(p, \Gamma)$ and $d(p, \Gamma)-\delta$ without intersecting $\Gamma$ that connects $p$ to one of $N_{i}^{\prime}$ or $N_{i}^{\prime \prime}$, as Figure 4 shown.

Therefore $\mathbb{R}^{2} \backslash \Gamma$ has at most two components.

2) $\mathbb{R}^{2} \backslash \Gamma$ has at least two components.

We place $\Gamma$ in the coordinate system so that for vertices $\left\{v_{i}=\left(x_{i}, y_{i}\right) \mid i=1, \cdots, n\right\}$, all $x_{i}$ are different. For each vertex $v_{i}$, we draw a vertical line $l_{i}$ through $v_{i}$, and notice that each such $l_{i}$ passes through exactly one vertex, namely, $v_{i}$ (Figure 5).

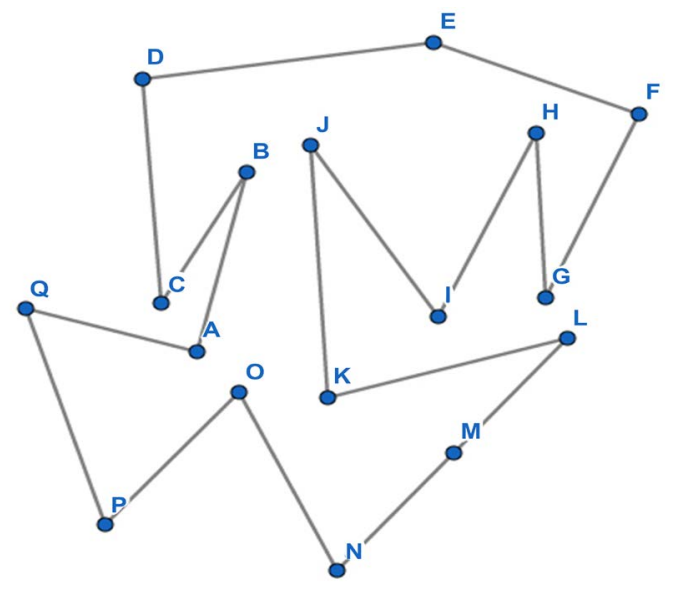

Figure 1. An example of Jordan polygon. Letters are vertices. Particularly, there exist letters, as the point $M$ suggests, that might be on the edge, which shows the case that the neighbouring edges of the very point are on the same line. 


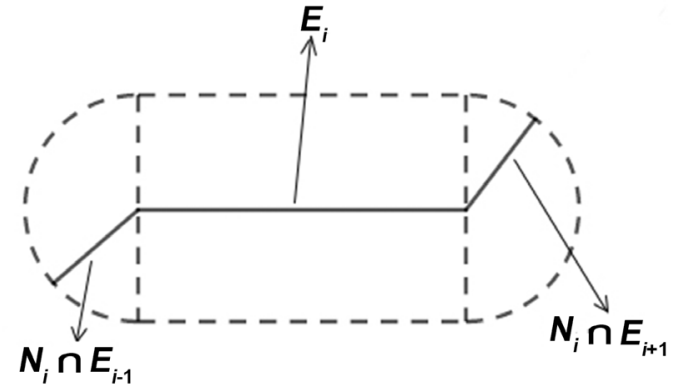

Figure 2. $N_{i}$ consists of a rectangle and two semicircles on opposite edges. It sometimes refers to a sausage domain.

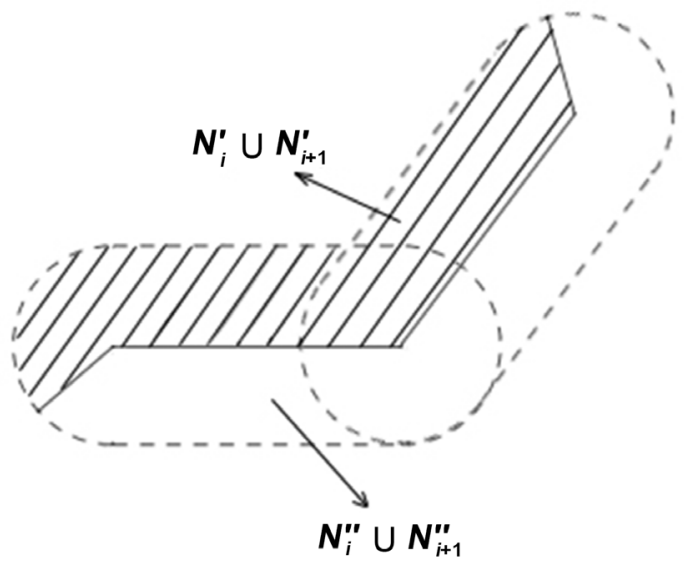

Figure 3. Edges of Jordan polygon partition $N_{i} \cup N_{i+1}$ into two connected components.

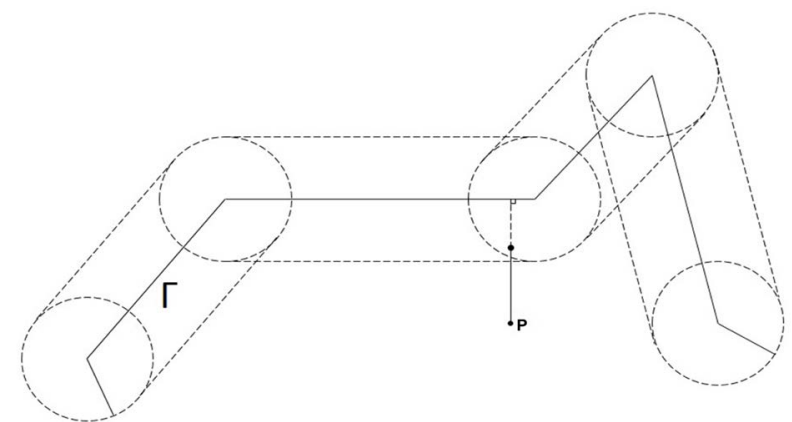

Figure 4. A line segment connecting $p$ to some $N_{i}$.

We say a line $l_{i}$ drawn as above is of type 1 if $v_{i-1}$ and $v_{i+1}$ are in opposite sides of $l_{i}$, i.e., $\left(x_{i-1}-x_{i}\right)\left(x_{i+1}-x_{i}\right)<0$, and of type 2 if $v_{i-1}$ and $v_{i+1}$ are in same side of $l_{i}$, i.e., $\left(x_{i-1}-x_{i}\right)\left(x_{i+1}-x_{i}\right)>0$. We say a vertex $v_{i}$ is of type 1 or 2 if the corresponding $l_{i}$ is of type 1 or 2 respectively. Observe that every $l_{i}$ is of either type 1 or type 2 . The same is true for $v_{i}$ 's.

We now partition $\mathbb{R}^{2} \backslash \Gamma$ into odd and even points and show that no continuous path connects an odd point to an even point.

For every point $p=(x(p), y(p))$ in $\mathbb{R}^{2} \backslash \Gamma$ we let $l_{p}$ be the upward vertical ray from $p$ and $m(p)$ be the number of intersection between $\Gamma$ and $l_{p}$, with the provision that if the $l_{p}$ passes some 


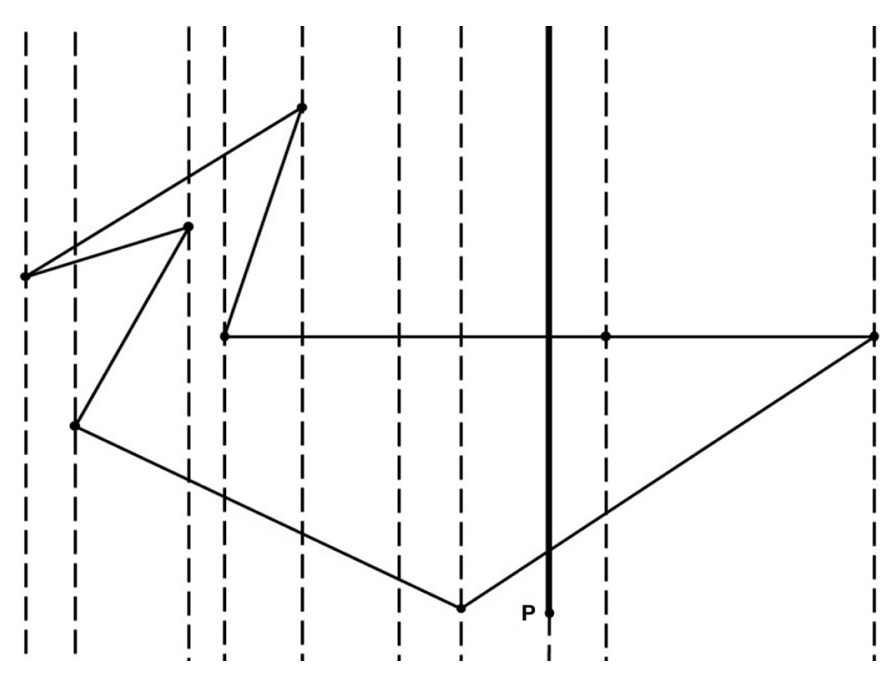

Figure 5. Type 1 or 2 of vertical lines through each vertex, plus a point $p$ with $m(p)=2$.

vertex $v_{i}$ of type 2 (there is only one such, by our choice of coordinate), then the intersection with $v_{i}$ is not counted into $m(p)$.

None of edges $E_{i}$ in $\Gamma$ is vertical hence $m(p) \in \mathbb{N}$ for every $p$. We call a point $p \in \mathbb{R}^{2} \backslash \Gamma$ is odd if $m(p)$ is odd, and is even if $m(p)$ is even. This is clearly a partition of $\mathbb{R}^{2} \backslash \Gamma$.

For every $p \in \mathbb{R}^{2} \backslash \Gamma$ there is an $\epsilon>0$ so that $p$ and $q$ are in the same parity whenever $d(p, q)<\epsilon$. To see this, we temporarily let $\Gamma_{1}=\Gamma \bigcup \bigcup_{i=1}^{n} l_{i}$ and notice that for every $p \in \mathbb{R}^{2} \backslash \Gamma$, either

1) $p \in \mathbb{R}^{2} \backslash \Gamma_{1}$; or

2) $p \in l_{i}$ for some $i, l_{p}$ passes a vertex $v_{i}$ of type 1 ; or

3) $p \in l_{i}$ for some $i, l_{p}$ passes a vertex $v_{i}$ of type 2 ; or

4) $p \in l_{i}$ for some $i$, but $l_{p}$ does not pass through any vertex.

For case (1) we take $\epsilon$ to be the radius of the open ball centered at $p$ without intersecting $\Gamma_{1}$, For cases (2) and (4) take $\epsilon$ be the radius of the open ball centered at $p$ intersects $\Gamma_{1}$ on $l_{i}$ only, For case (3) we can find an open ball centered at $p$ with radius $\epsilon$ intersecting $\Gamma_{1}$ on $l_{i}$ only, as in case (2) or (4), and by our definition of $m(p)$, any $q$ in the open ball has $m(q)=m(p)$ or $m(p)+2$ (Figure 6).

We have shown that every point sufficiently close to an odd (resp.even) point is odd (resp.even). If $\Pi:[0,1] \rightarrow \mathbb{R}^{2} \backslash \Gamma$ is a continuous path with $\Pi(0)$ is odd and $\Pi(1)$ is even, let

$$
t_{0}:=\inf (\{t \in[0,1] \mid \Pi(t) \text { is even }\})
$$

then $t_{0}>0$ and for some $\epsilon>0$ we have $\Pi(t)$ is odd for all $t_{0}-\epsilon<t<t_{0}$, while $\Pi(t)$ is even for all $t_{0}<t<t_{0}+\epsilon$. By continuity of $\Pi, \Pi\left(t_{0}\right)$ would be neither odd or even, which is absurd (Figure 7).

It remains to show that there do exist even points and odd points.

Take any $p$ so that $m(p) \neq 0$, then there is an open ball $B$ centred at the last (or, highest) intersection between $\Gamma$ and $l_{p}$ that counted into $m(p)$, which contains only one connected component of $\Gamma$ (namely, the component where our intersection lies in). $B$ is divided into upper half and lower half by that component of $\Gamma$. Any point $p_{0}$ of $l_{p}$ in the lower half of $B$ will have $m\left(p_{0}\right)=1$ hence $p_{0}$ is odd.

Take a sufficiently large open ball $B^{\prime} \in \mathbb{R}^{2}$ covering $\Gamma$, any point $q$ outside $B^{\prime}$ would have $m(q)=0$ hence $q$ is even (Figure 8).

We have shown that $\mathbb{R}^{2} \backslash \Gamma$ has indeed two connected components. Therefore the proof of Lemma 1 is complete.

Remark. The above proof has shown that a Jordan polygon divides the plane into a bounded connected component $O$ and an unbounded connected component $V$. Moreover, $O$ and $V$ are nonempty and open. 


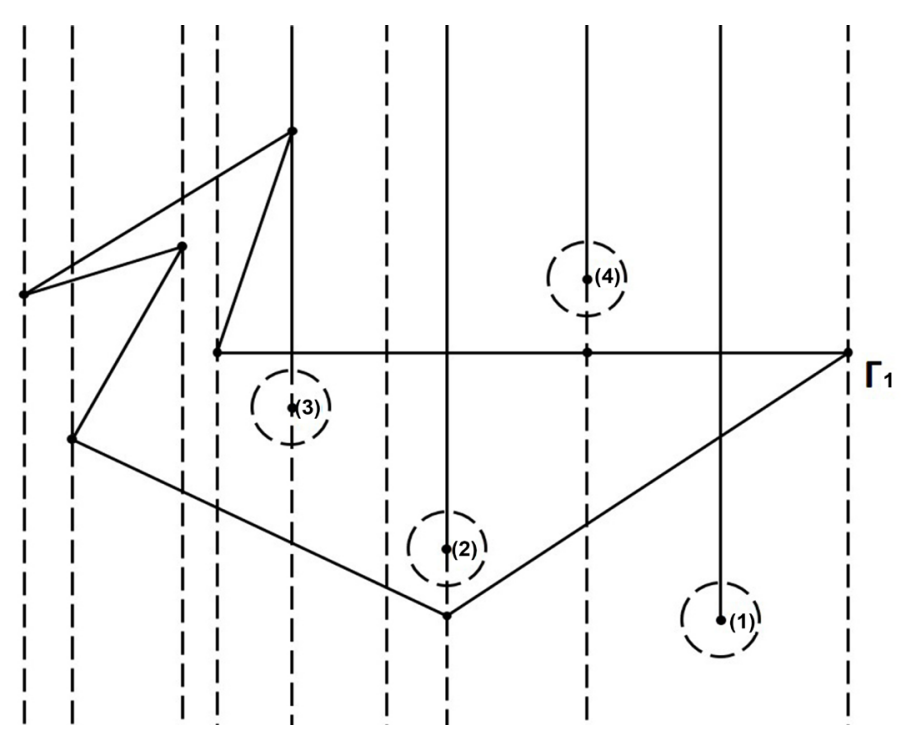

Figure 6. Points in $\mathbb{R}^{2} \backslash \Gamma$ belong to four different cases.

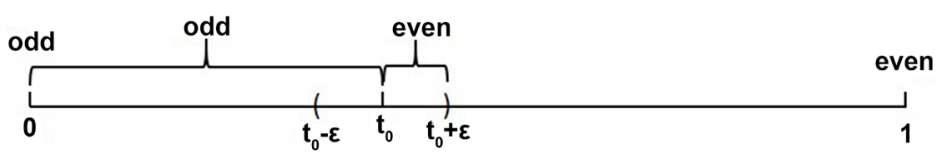

Figure 7. Behaviour of $\Pi$ around $t_{0}$.

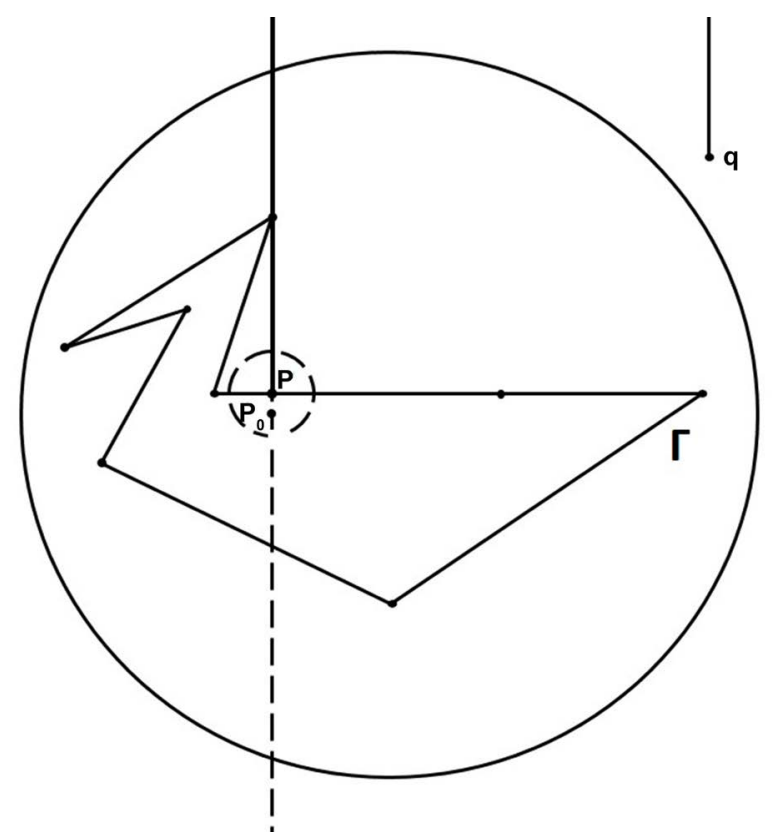

Figure 8. An odd point $p_{0}$ and an even point $q$.

Next we show that any Jordan curve $\Gamma$ could be approximated uniformly by a sequence of Jordan polygons $\left(\Gamma_{n}\right)$.

Lemma 2. Every Jordan Curve $\Gamma$ with parametrisation $\gamma$ could be approximated arbitrarily well by a Jordan polygon $\Gamma^{\prime}$ with parametrisation $\gamma^{\prime}$. Moreover, it should be noted, the vertices on $\Gamma^{\prime}$ all lie on $\Gamma$. 
Proof. We want to show that given any $\epsilon>0$, there is some Jordan polygon $\Gamma^{\prime}$ so that $\left|\gamma(\cos \theta, \sin \theta)-\gamma^{\prime}(\cos \theta, \sin \theta)\right|<\frac{\epsilon}{2}, \quad \forall(\cos \theta, \sin \theta) \in C$

By uniform continuity we can choose $\epsilon_{1}>0$ such that

$$
|p-q| \leq \epsilon_{1} \Rightarrow|\gamma(p)-\gamma(q)|<\frac{\epsilon}{2}[6]
$$

and $\epsilon_{2}>0$ such that

$$
|\gamma(p)-\gamma(q)| \leq \epsilon_{2} \Rightarrow|p-q|<\min \left(\epsilon_{1}, \sqrt{3}\right) \quad[6]
$$

Put $\delta=\min \left(\frac{\epsilon}{2}, \epsilon_{2}\right)$

If we fill the plane by squares with vertices $\left(k \frac{\delta}{\sqrt{2}}, l \frac{\delta}{\sqrt{2}}\right), k, l \in \mathbb{N}$, then the side length of each square is $\frac{\delta}{\sqrt{2}}$. And the distance between every pair of points in the same square, say $S$, is smaller than $\delta$, therefore either $\gamma^{-1}(S)=\varnothing$, or

$$
\operatorname{diam}\left(\gamma^{-1}(S)\right)<\min \left(\epsilon_{1}, \sqrt{3}\right)[6]
$$

Each $\gamma^{-1}(S)$ is contained in a unique minimal arc $A$ on $C$, with radian shorter than $\frac{2 \pi}{3}$ (Figure 9).

We now construct $\Gamma^{\prime}$ stated in the lemma in a finite process. Observe that $\Gamma$ meets only finitely many of the squares, we label them $S_{1}, S_{2}, \cdots, S_{n}$.

We first change $\Gamma_{0}=\Gamma$ into another Jordan curve $\Gamma_{1}$. Let $A_{1}$ be the unique minimal circular arc on $C$ containing $\gamma_{0}^{-1}\left(S_{1}\right)\left(=\gamma^{-1}\left(S_{1}\right)\right)$. Define $\gamma_{1}=\gamma_{0} \quad(=\gamma)$ outside $A_{1}$, and for $(\cos \theta, \sin \theta) \in A_{1}$, $\gamma_{1}(\cos \theta, \sin \theta)=\left(a_{1} \theta+b_{1}, c_{1} \theta+d_{1}\right)$, where $a_{1}, b_{1}, c_{1}, d_{1}$ are chosen so that $\gamma_{1}$ is continuous on $C$. Note that when $i \geq 2, \gamma_{1}^{-1}\left(S_{i}\right) \subseteq \gamma_{0}^{-1}\left(S_{i}\right)$, and thus has diameter less than $\sqrt{3}$; it could even be empty (Figures 10-12).

The procedure would end in at most $n$ steps as $\Gamma$ intersects only $n$ squares. We claim that $\Gamma_{n}$ is our desired $\Gamma^{\prime}$ (Figure 13).

By above argument each circular arc $A_{j}$ on $C$ containing $\gamma_{j-1}^{-1}\left(S_{j}\right)$, if nonempty, has radian less than $\frac{2 \pi}{3}$. Consider now any $a \in C$ for which $\gamma_{n}(a) \neq \gamma(a)$. There is $i$ so that $\gamma_{n}(a)=\gamma_{i}(a) \neq \gamma_{i-1}(a)$. By construction a belongs to the arc $A_{i}$, with endpoints $b, c$, where $\gamma(b), \gamma(c)$ in the same square $S_{i}$, say. By above procedure again we have $\gamma_{i}(b)=\gamma(b), \gamma_{i}(c)=\gamma(c)$. Then

$$
\begin{aligned}
\left|\gamma(a)-\gamma_{n}(a)\right| & =\left|\gamma(a)-\gamma(b)+\gamma_{i}(b)-\gamma_{i}(a)\right| \\
& \leq|\gamma(a)-\gamma(b)|+\delta \\
& \leq|\gamma(a)-\gamma(b)|+\frac{\epsilon}{2}
\end{aligned}
$$

Because $|\gamma(b)-\gamma(c)| \leq \delta \leq \epsilon_{2} \Rightarrow|b-c| \leq \epsilon_{1}$ and $|a-b| \leq|b-c|$, we have $|a-b| \leq \epsilon_{1}$. Therefore $|\gamma(a)-\gamma(b)|<\frac{\epsilon}{2}$, showing that $\left|\gamma(a)-\gamma_{n}(a)\right|<\epsilon$ 


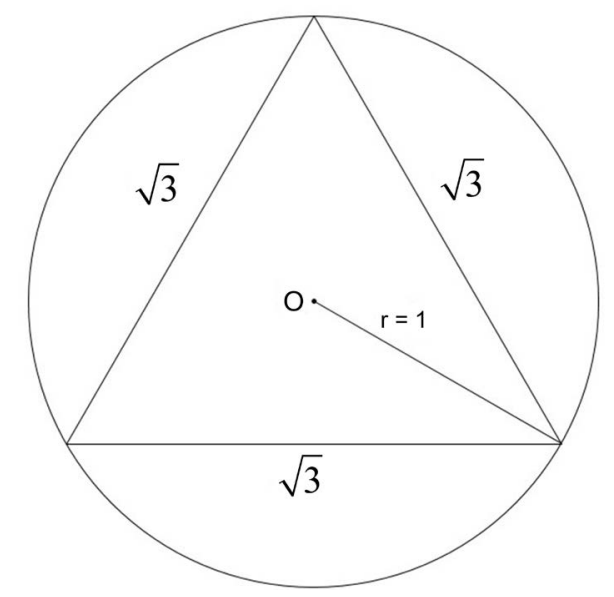

Figure 9. The inscribed equilateral triangle of a circle. The triangle has side length equals to $\sqrt{3}$. By restricting the diameter of inverse image of each square to be less than $\sqrt{3}$, the Jordan curve meets at least three different squares so the approximation would not degenerate to a single line segment. (replace $\sqrt{3}$ by the side length of any inscribed regular polygon in a circle should also work.)
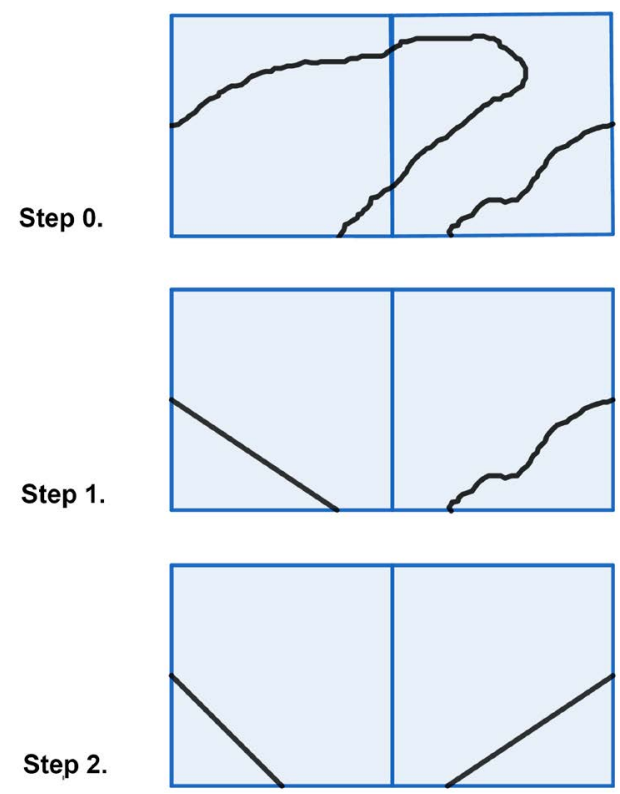

Figure 10. Having produced $\Gamma_{i-1}$, let $A_{i}$ be the unique minimal arc on $C$ containing $\gamma_{i-1}^{-1}\left(S_{i}\right)$. If $\gamma_{i-1}^{-1}\left(S_{i}\right)=\varnothing$ then let $A_{i}:=\varnothing$ and $\Gamma_{i}:=\Gamma_{i-1}$. Otherwise define $\gamma_{i}:=\gamma_{i-1}$ outside $A_{i}$, and for $(\cos \theta, \sin \theta) \in A_{i}$, $\gamma_{i}(\cos \theta, \sin \theta):=\left(a_{i} \theta+b_{i}, c_{i} \theta+d_{i}\right)$, where $a_{i}, b_{i}, c_{i}, d_{i}$ are chosen so that $\gamma_{i}$ is continuous on $C$. 


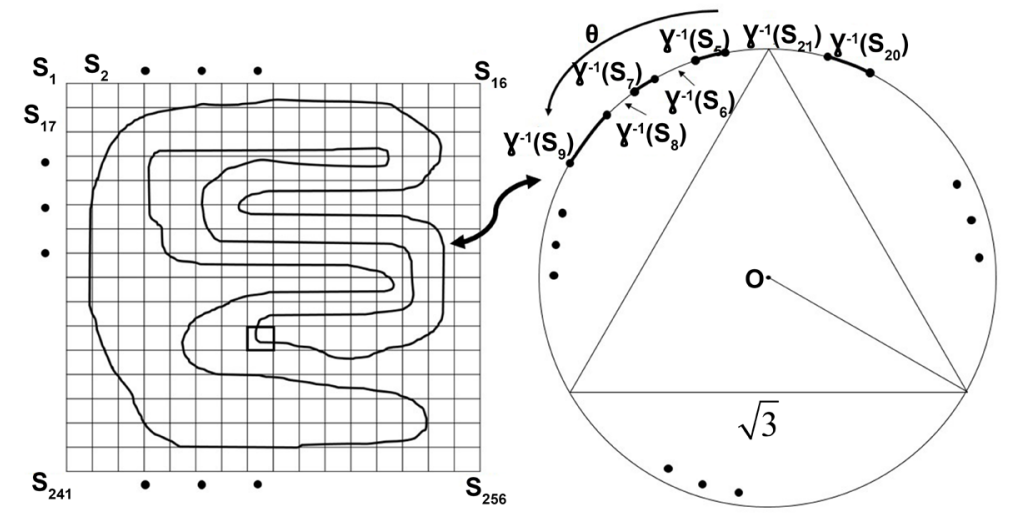

Figure 11. Each square corresponds to an arc on the parametrising circle $\mathrm{C}$.

$\mathbf{S}_{168}$

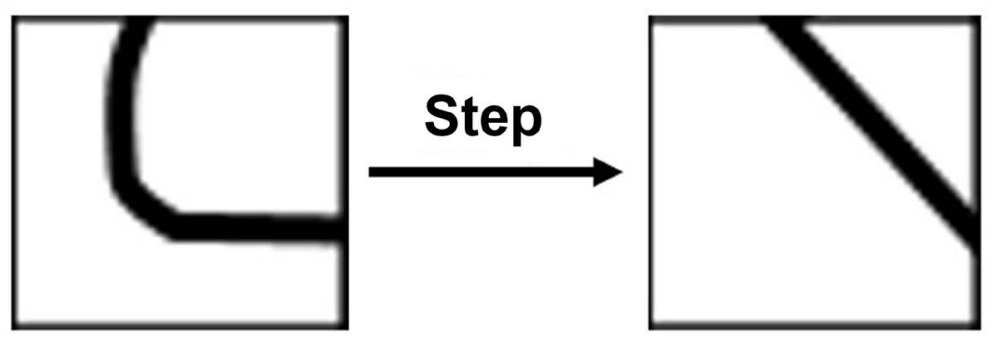

Figure 12. The action on square S168.

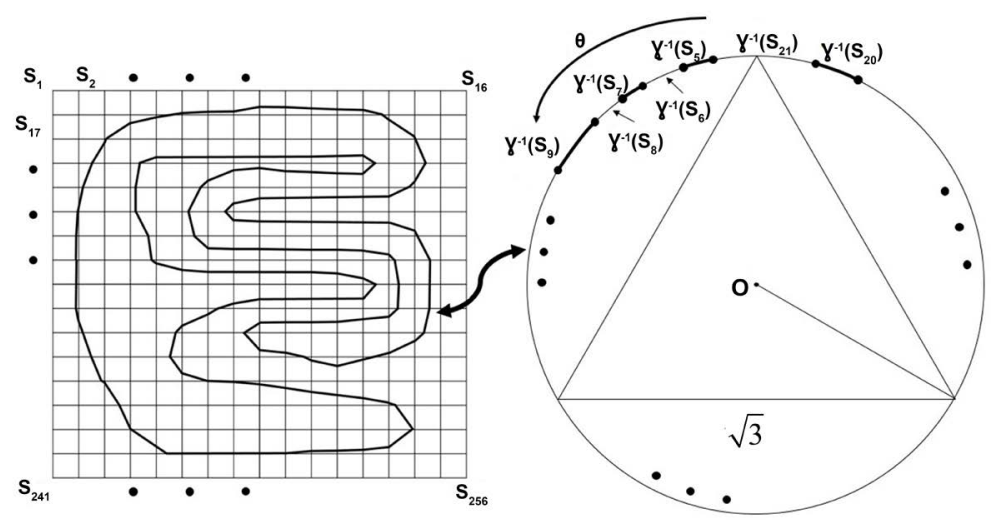

Figure 13. Polygonal approximation for curve in Figure 11.

Remark. Notice the two points on the unit circle $C$ of distance $\sqrt{3}$ apart correspond to endpoints of an arc of radian $\frac{2 \pi}{3}$. This radian produced an inscribed equilateral triangle which is the "smallest" regular polygon one could produce on the unit circle. it helps us, in the above process, really produce a polygon for each $\Gamma_{i}$.

We can now approximate $\Gamma$ by taking (uniform) limit of a sequence of Jordan polygons $\left(\Gamma_{n}\right)$. To show JCT holds for general $\Gamma$ we need to eliminate cases that 
a) $\mathbb{R}^{2} \backslash \Gamma=\mathbb{R}^{2} \backslash\left(\lim _{n \rightarrow \infty} \Gamma_{n}\right)$ has no bounded connected component while each $\mathbb{R}^{2} \backslash \Gamma_{n}$ has;

b) $\mathbb{R}^{2} \backslash \Gamma$ has more than two bounded components.

The following lemmas 3 and 4 ensure that (a) and (b) mentioned above would not happen in the limiting process.

For every point $p \in O$ where $O$ is the open bounded component enclosed by a Jordan polygon, we claim that there exists a circle in $O$ containing $p$ that meets $\Gamma$ in more than one point. There is an open disc $D$ centred at $p$ inside $O$. We enlarge the radius of $D$ until boundary of $D$ meets $\Gamma$. If they meet in more than one point we are done. Otherwise suppose $\gamma(a)$ is the only intersection, we consider a variable circle through $\gamma(a)$, with center moves from $p$ in the direction $\overrightarrow{\gamma(a) p}$ until it meets $\Gamma$ in another point $\gamma(b) . a$ and $b$ are on the unit circle $C$ thus $|a-b|$ is bounded. By Bolzano-Weierestrass there exists a disc $D$ as described with $|a-b|$ maximal (Figure 14).

Lemma 3. Let $\Gamma$ be a Jordan polygon. Then the bounded component of $\mathbb{R}^{2} \backslash \Gamma$ contains a disc, on the boundary circle of which are two points $\gamma(a)$ and $\gamma(b)$, with $|a-b| \geq \sqrt{3}$.

Proof. The existence of the disc $D$ with $|a-b|$ maximal is clear from above discussion. Assume $|a-b|<\sqrt{3}$. Then $a$ and $b$ are the endpoints of an $\operatorname{arc} A$ of radian $>\frac{4 \pi}{3}$. The boundary circle cannot meet $\gamma(A) \backslash\{\gamma(a), \gamma(b)\}$ as $\max \{|a-c|,|b-c|\}>|a-b|$ for every $c$ in $A \backslash\{a, b\}$ (Figure 15).

Let $\gamma\left(v_{1}\right), \cdots, \gamma\left(v_{n}\right)$ be the vertices of $\Gamma$ on $\gamma(A)$ as met when passing from $\gamma(a)$ to $\gamma(b)$. By Lemma 1 , the chord $\gamma(a) \gamma(b)$ divides the interior of Jordan polygon into two parts, say $P$ and $Q$. We have $\gamma\left(v_{1}\right), \cdots, \gamma\left(v_{n}\right)$ are in the same component, say $P$. We claim that $\gamma\left(v_{1}\right)$ and $\gamma\left(v_{n}\right)$ are in the same side of the $\gamma(a) \gamma(b)$ (Figure 16).

Suppose $\gamma\left(v_{n}\right)$ and $\gamma\left(v_{1}\right)$ are in opposite sides of $\gamma(a) \gamma(b)$. Notice that there is no vertex between $\gamma(a)$ and $\gamma\left(v_{1}\right)$, neither between $\gamma(b)$ and $\gamma\left(v_{n}\right)$. From the proof in Lemma 1, we can find $\delta>0$ such that any open ball centred at a point in $\Gamma \bigcup \gamma(a) \gamma(b)$ intersects $\Gamma \bigcup \gamma(a) \gamma(b)$ on a single connected component of $\Gamma$. Take $\alpha, \beta \in P$ such that $d\left(\gamma\left(v_{1}\right), \alpha\right)<\delta$ and that $d\left(\gamma\left(v_{n}\right), \beta\right)<\delta$. Let $\pi_{1}$ be a path in $P$ from $\alpha$ to $\beta, \pi_{2}$ the path from $\alpha$ to $\alpha^{\prime}$ a point with distance to the midpoint of $\gamma(a) \gamma(b)$ less than $\delta$, similarly, $\pi_{3}$ the path from $\beta$ to $\beta^{\prime}$ a point with distance to the midpoint of $\gamma(a) \gamma(b)$ less than $\delta$, notice that $\alpha^{\prime}, \beta^{\prime}$ are in different sides of $\gamma(a) \gamma(b)$. But this leads to a contradiction: $\gamma(a) \gamma(b)$ doesn't separate the interior of $\Gamma$.

Now $a$ and $b$ could either belong to those vertices or not. We shall divide the situation into different cases (Figure 17).

1) $a \neq v_{1}$ and $b \neq v_{n}$.

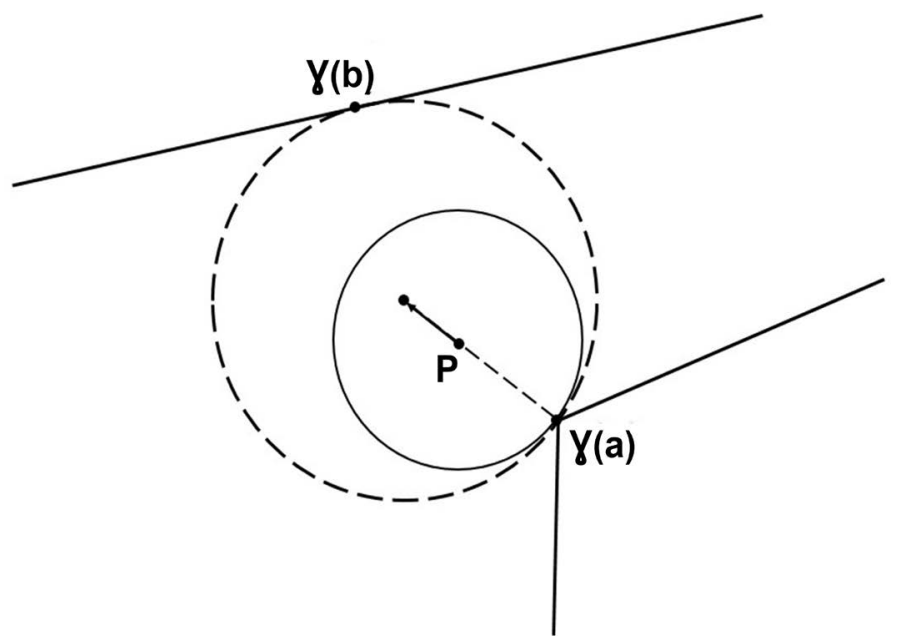

Figure 14. There is always a circle containing $p$ meets

$\Gamma$ at two points. 


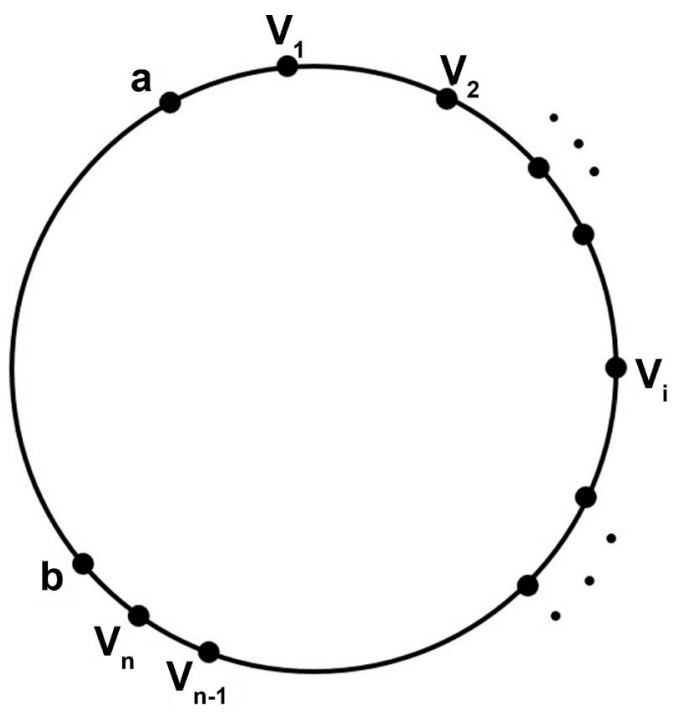

Figure 15. Positions on $A$ of vertices as met when moving from $\gamma(a)$ to $\gamma(b)$.

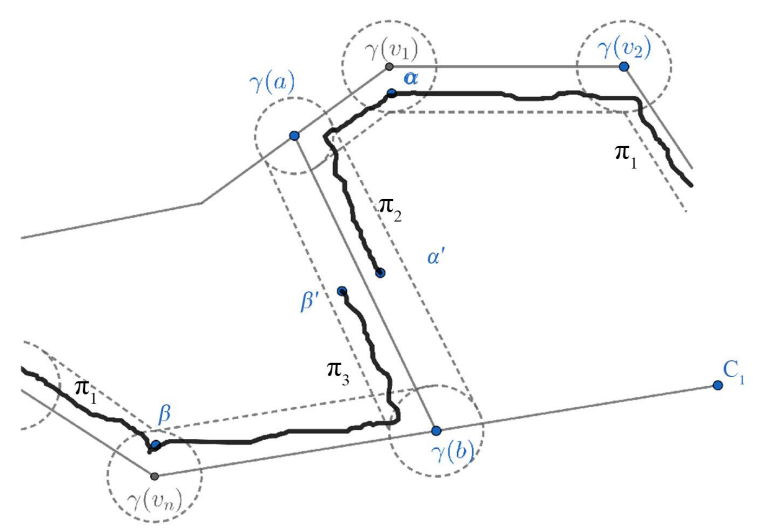

Figure 16. The path $\pi_{2} \cup \pi_{1} \bigcup \pi_{3}$ in $P$ connecting $\alpha^{\prime}$ and $\beta^{\prime}$ in both sides of $\gamma(a) \gamma(b)$.

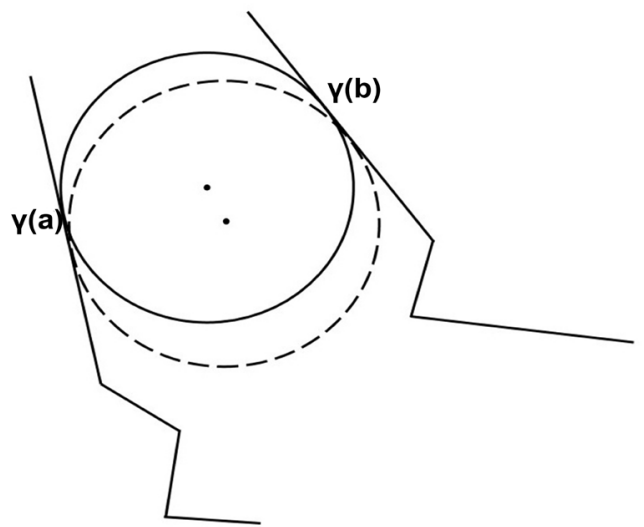

Figure 17. Case 1. A circle touching $\Gamma$ at points very close to $\gamma(\boldsymbol{a})$ and $\gamma(\boldsymbol{b})$. 


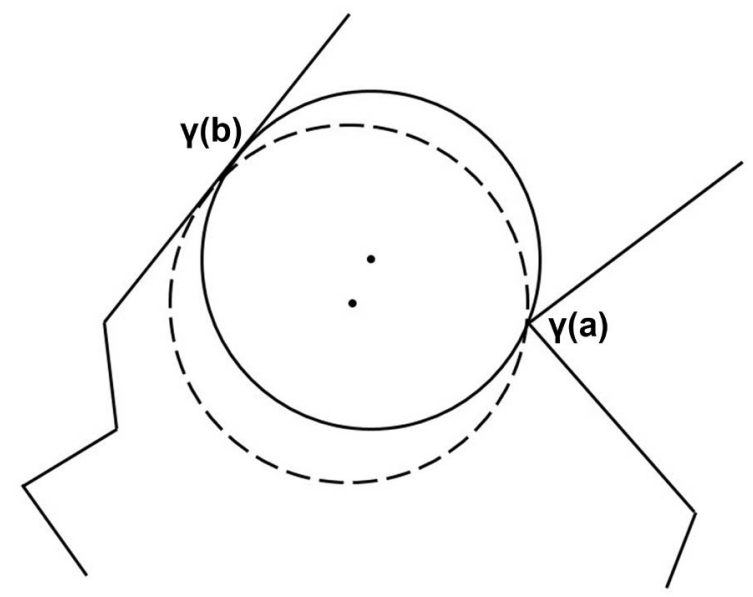

\section{Figure 18. Case 2. A circle passing $\gamma(a)$ and touching $\Gamma$ at a point very close to $\gamma(b)$.}

In this case the boundary of $D$ is tangent to both $\gamma(a) \gamma\left(v_{1}\right)$ and $\gamma(b) \gamma\left(v_{n}\right)$. Consider a circle touching segment $\gamma(a) \gamma\left(v_{1}\right)$ at a point $\gamma\left(a^{\prime}\right)$ very near $\gamma(a)$ and touching segment $\gamma(b) \gamma\left(v_{n}\right)$ at a point $\gamma\left(b^{\prime}\right)$ very near $\gamma(b)$, where $a^{\prime}, b^{\prime} \in A$. Provided they are close enough, the now circle would meet $\Gamma$ in those two points only. As $\left|a^{\prime}-b^{\prime}\right|>|a-b|$ contradiction arises (Figure 18).

2) $a=v_{1}$ and $b \neq v_{n}$.

The boundary of $D$ passes $\gamma(a)$ and is tangent to $\gamma(b) \gamma\left(v_{n}\right)$. We choose a circle passing $\gamma(a)$ and touching $\gamma(b) \gamma\left(v_{n}\right)$ at a point $\gamma\left(b^{\prime}\right)$ very close to $\gamma(b)$ where $b^{\prime} \in A$. A contradiction similar to case (1) arises (Figure 19).

3) $a=v_{1}$ and $b=v_{n}$.

We consider a variable circle through $\gamma(a)$ and $\gamma(b)$ and move its center from center of $D$ to the domain bounded by the radii of $D$ to $\gamma(a)$ and $\gamma(b)$ together with $\gamma(A)$. The circle will eventually either

- meet $\Gamma$ at some point $\gamma(c) \in \gamma(A) \backslash\{\gamma(a), \gamma(b)\}$ contradict to maximality of $|a-b|$ as $\max \{|a-c|,|b-c|\}>|a-b|$ for every $c$ in $A \backslash\{a, b\}$; or

- become tangent to segment $\gamma(a) \gamma\left(v_{2}\right)$ or $\gamma(b) \gamma\left(v_{n-1}\right)$ reduced to cases (1) or (2).

Consider $\Gamma$ a Jordan polygon in $\mathbb{R}^{2}$ which divides the plane into two components. Let $X$ be one of those components. By a chord $S$ in $X$ we mean a line segment in $X$ (except for its endpoints) between two distinct points on $\Gamma$. By Lemma 1, $X \backslash S$ consists of two connected open sets. Fix two points $a$ and $b$, suppose $d(\Gamma,\{a, b\}) \geq 1$, and assume that for every $S$ of length less than 2 , $a$ and $b$ are in the same component of $X \backslash S$. Then we have:

Lemma 4. Under the assumptions stated there is a continuous path $\Pi$ from $a$ to $b$ such that $d(\Pi, \Gamma) \geq 1$.

Proof. We first note that if $a^{\prime}$ is any point in $\mathbb{R}^{2}$, connected to a by a continuous path $\Pi^{\prime}$, where $d\left(\Pi^{\prime}, \Gamma\right) \geq 1$. If $S$ is any chord of length less than $2, \Pi^{\prime}$ would not pass $S$ so a and $a^{\prime}$ are in the same component of $X \backslash S$. By assumption $a$ and $b$ are in the same component, so are $a^{\prime}$ and $b$.

Hence we may assume now that $d(a, \Gamma)=d(b, \Gamma)=1$.

Choose $\gamma\left(u_{a}\right)$ and $\gamma\left(u_{b}\right)$ on $\Gamma$ (so $\left.u_{a}, u_{b} \in C\right)$. Let $D$ be a mobile circle initially placed with its center $c$ in $a$. We roll the circle along $\Gamma$ starting at $\gamma\left(u_{a}\right)$ until $c$ falls in $b$. The desired path $\Pi$ will be obtained as the curve followed by $c$.

$D$ arrives at $\gamma\left(u_{b}\right)$.

Suppose not. At some position, $D$ and $\Gamma$ has some common chord $S=\gamma\left(u_{1}\right) \gamma\left(u_{2}\right)$ of length less than 2 (see Figure 20). $X \backslash S$ consists of two components, say $Y$ and $Z$. By assumption $a$ and $b$ are in the 


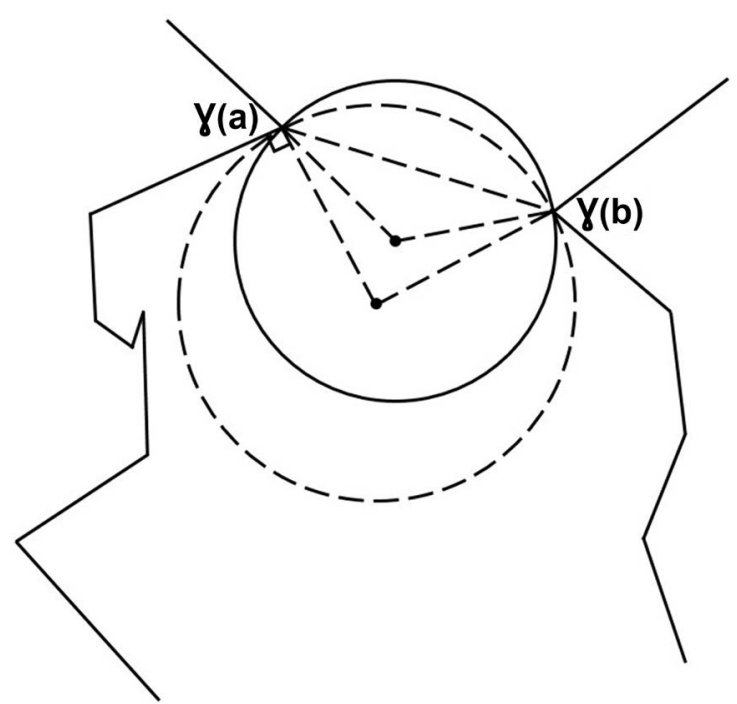

Figure 19. Case 3. As the variable circle passing two fixed points $\gamma(a)$ and $\gamma(b)$ while its center moves away, it will either meet the third point of $\Gamma$, which contradicts our construction, or it will eventually tangent to $\gamma(a) \gamma\left(v_{1}\right)$ or $\gamma(b) \gamma\left(v_{n}\right)$ or both.

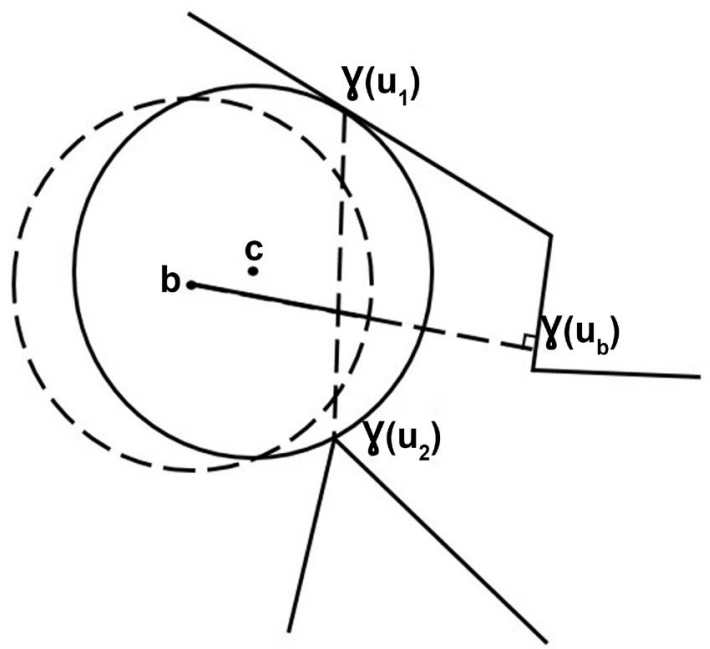

Figure 20. The radius of $E$ towards $\gamma\left(u_{b}\right)$ would never meet $\gamma\left(u_{b}\right)$. Note that $E$ meets $\gamma\left(u_{1}\right) \gamma\left(u_{2}\right)$ at two points.

same component, say $Y$, replacing $a^{\prime}$ by $c$ in the first paragraph $c$ is also in $Y$. For $D$ doesn't reach $\gamma\left(u_{b}\right)$, $\gamma\left(u_{b}\right)$ must lie in the boundary of $Z$, which is between $\gamma\left(u_{1}\right)$ and $\gamma\left(u_{2}\right)$ on $\Gamma$.

Now draw a unit circle $E$ centred at $b$. The circle meets $\Gamma$ at $\gamma\left(u_{b}\right)$. Draw the radius of $E$ to $\gamma\left(u_{b}\right)$. Since $b$ and $\gamma\left(u_{b}\right)$ are in the opposite sides of $S$, the radius crosses $S$.

As $E$ lies in $X$, and $E \neq D, E$ encompasses neither $\gamma\left(u_{1}\right)$ nor $\gamma\left(u_{2}\right)$ while $D$ does. So $E$ intersects $S$ at two points. Now $b$ and $c$ lie to the same side of $S$, with $d(b, S)>d(c, S)$ and thus $d(b, Z)>(c, Z)$. The radius of $E$ from $b$ to $\gamma\left(u_{b}\right)$ must fall inside or on $D$ so can never reach $\gamma\left(u_{b}\right)$, which is absurd. 
$c$ arrives at $b$

We have verified that $D$ reaches $\gamma\left(u_{b}\right)$, a point on $\Gamma$ of distance 1 away from $b$. We have to show that at this position, $c$ is in $b$.

The statement is clear when $\gamma\left(u_{b}\right)$ is not a vertex. The problem happens when $\gamma\left(u_{b}\right)$ is a vertex, and there is a common chord $S=\gamma\left(u_{b}\right) y$ of length less than 2 between $c$ and $b$, where $y$ is some point on $\Gamma$ (see Figure 21).

\section{PROOF OF JORDAN'S THEOREM}

\section{$\mathbb{R}^{2} \backslash \Gamma$ has at least two components:}

The existence of unbounded component is clear. For the bounded one, draw a large closed disc $D$ with boundary circle $C_{0}$ around $\Gamma$, let $\Gamma_{1}, \Gamma_{2}, \cdots$ be a sequence of Jordan polygons converging uniformly to $\Gamma$. We may assume that all $\Gamma_{n}$ lie inside $C_{0}$. By Lemma 3, there is a circle $C_{n}$ centred $z_{n}$ in the bounded component on $\mathbb{R}^{2} \backslash \Gamma_{n}$ so that there are points $\gamma_{n}\left(a_{n}\right), \gamma_{n}\left(b_{n}\right) \in \Gamma_{n} \cap C_{n}$ with $\left|a_{n}-b_{n}\right| \geq \sqrt{3}$. By Bolzano-Weierstrass theorem again there is a limit point, say $z$, in $D$. Passing to a subsequence of the original one we may assume that $Z_{n} \rightarrow Z$ as $n \rightarrow \infty$.

By uniform continuity there exists $\epsilon>0$ such that

$$
|a-b| \geq \sqrt{3} \Rightarrow|\gamma(a)-\gamma(b)| \geq \epsilon
$$
that

By our choice of $\left(a_{n}, b_{n}\right)$ we have $\left|\gamma\left(a_{n}\right)-\gamma\left(b_{n}\right)\right| \geq \epsilon$. since $\gamma_{n} \rightarrow \gamma$, There exists $N_{1} \in \mathbb{N}$ such

$$
\left|\gamma\left(a_{n}\right)-\gamma\left(b_{n}\right)\right|>\frac{\epsilon}{2} \text { if } n>N_{1}
$$

Therefore $\operatorname{diam}\left(C_{n}\right)>\frac{\epsilon}{2}$, so $d\left(z_{n}, \Gamma_{n}\right)>\frac{\epsilon}{4}$.

Since $z_{n} \rightarrow Z$ we also have $N_{2} \in \mathbb{N}$

$$
d\left(z, z_{n}\right)=\left|z-z_{n}\right|<\frac{\epsilon}{8}
$$

Taking $N:=\max \left\{N_{1}, N_{2}\right\}$ we have for $n>N$

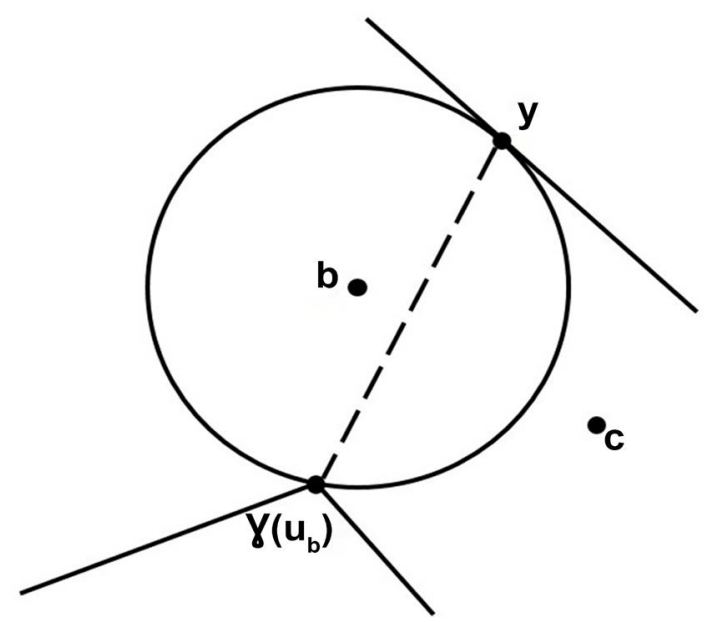

Figure 21. But this is not possible: again replacing $a^{\prime}$ in the first paragraph by $c$ we have $a, b$, and $c$ are in the same component of $X \backslash S$, so $b$ and $c$ should be in the same side. 


$$
d\left(z, \Gamma_{n}\right) \geq d\left(\Gamma_{n}, z_{n}\right)-d\left(z_{n}, z\right) \geq \frac{\epsilon}{8}
$$

so $z$ and $Z_{n}$ are in the same component of $\mathbb{R}^{2} \backslash \Gamma_{n}$. The same is true for $\mathbb{R}^{2} \backslash \Gamma$.

If $z$ is in the unbounded component of $\mathbb{R}^{2} \backslash \Gamma$, we could find a continuous path $\Pi$ from $z$ to a point outside $C_{0}$. Set $\delta=d(\Pi, \Gamma)$. Again by our choice of $\left(\Gamma_{n}\right)$, there exists $N_{1} \in \mathbb{N}$ such that for $n>N_{1}$,

$$
d\left(\gamma_{n}, \gamma\right)=\left|\gamma_{n}-\gamma\right|<\frac{\delta}{2}
$$

so for such $n$,

$$
d\left(z, \Gamma_{n}\right) \geq d\left(\Pi, \Gamma_{n}\right)>\frac{\delta}{2}
$$

and some $N_{2} \in \mathbb{N}$ such that for $n>N_{2}$,

$$
d\left(z_{n}, z\right)=\left|z-z_{n}\right|<\frac{\delta}{2}
$$

Taking $N>\max \left\{N_{1}, N_{2}\right\}$, we have $d\left(z, z_{n}\right) \leq d\left(z, \Gamma_{n}\right)$ so $z_{n}$ and $z$ are both in the unbounded component of $\mathbb{R}^{2} \backslash \Gamma_{n}$, which contradicts to the definition of $z_{n}$.

$\mathbb{R}^{2} \backslash \Gamma$ has at most two components: Suppose not. Let $p, q, r \in \mathbb{R}^{2} \backslash \Gamma$ be points from three distinct components of $\mathbb{R}^{2} \backslash \Gamma$, with $d(\Gamma,\{p, q, r\})=\epsilon$. Let $\Gamma_{1}, \Gamma_{2}, \cdots \rightarrow \Gamma$ uniformly as we did. Then $d\left(\Gamma_{n},\{p, q, r\}\right) \geq \frac{\epsilon}{2}$.

For any $n \in \mathbb{N}$, two of the three points should be in the same component $X_{n}$ of $\mathbb{R}^{2} \backslash \Gamma$. By passing to a subsequence we may assume that $p$ and $q$ are in $X_{n}$ for all $n$.

Assume first that there is some $\delta \in(0, \epsilon)$ and infinitely many $n$ so that $p$ and $q$ are connected by a continuous path $\Pi_{n}$ with $d\left(\Pi_{n}, \Gamma_{n}\right) \geq \delta$, as $\Gamma_{n} \rightarrow \Gamma$, for large $n, d\left(\Gamma, \Pi_{n}\right)>\frac{\delta}{2}$, i.e., $p$ and $q$ could be connected by a path not intersecting $\Gamma$, which is not possible. Hence there is no such $\delta$.

By above argument we conclude that for any $\delta>0$ any path $\Pi$ connecting $p$ and $q$ would have $d\left(\Gamma_{n}, \Pi\right)<\delta$ for all but finitely many $\delta$. It yields, together with Lemma 4 , an increasing sequence $n_{1}, n_{2}, \cdots$, and a sequence of chords $S_{1} \subset X_{n_{1}}, S_{2} \subset X_{n_{2}}, \cdots$, so that one has:

1) $p$ and $q$ are in different components of $X_{n_{i}} \backslash S_{i}$;

2) $\left|\gamma_{n_{i}}\left(a_{i}\right)-\gamma_{n_{i}}\left(b_{i}\right)\right| \rightarrow 0$ as $i \rightarrow \infty$, where $\gamma_{n_{i}}\left(a_{i}\right)$ and $\gamma_{n_{i}}\left(b_{i}\right)$ are endpoints of $S_{i}$.

Let $T$ be the component of $X_{n_{i}} \backslash S_{i}$ bounded by $S_{i}$ and $\gamma_{n_{i}}\left(A_{i}\right)$ where $A_{i}$ is the smaller arc on $C$ with endpoints $a_{i}$ and $b_{i}$. Suppose, without loss of generality, $p \in T$. Now since $\gamma_{n} \rightarrow \gamma$ and $\gamma$ is homeomorphic, as $i \rightarrow \infty,\left|\gamma\left(a_{i}\right)-\gamma\left(b_{i}\right)\right| \rightarrow 0$, thus $\left|a_{i}-b_{i}\right| \rightarrow 0$ and therefore $\operatorname{diam}\left(\gamma_{n_{i}}\left(A_{i}\right)\right) \rightarrow 0$. For large $i$,

$$
\operatorname{diam}(T) \leq \operatorname{diam}\left(\gamma_{n_{i}}\left(A_{i}\right)\right)+\operatorname{diam}\left(S_{i}\right)<\epsilon
$$

In particular, we have $\left|p-\gamma\left(a_{i}\right)\right|<\epsilon$, contradicts to $d(\Gamma,\{p, q, r\})>\epsilon$.

\section{CONCLUSION}

So far the proof of Jordan Curve Theorem is done. The four lemmas are of paramount importance in the whole proof. Especially, the first lemma, which Jordan was in an inappropriate way of demonstration, seems to be intuitive, however, requires rigorous proof. This theorem is discussed in the $\mathbb{R}^{2}$ and it is a useful theorem to bolster the basis of the edifice in Topology.

\section{ACKNOWLEDGEMENTS}

I'm very grateful that the Professor Charles C. Pugh from the Department of Mathematics, University 
of California of Berkeley helped me a lot with my study of Topology. And I appreciate my groupmate Haiqi $\mathrm{Wu}$ from Oxford helped me with my paper. And thanks to the platform provided by CIS organization, I have the chance to study topology with the emeritus professor and hence accomplish the paper. Also if it were not for the teachers from my alma mater Wuhan University, I wouldn't have been able to walk on my avenue of Mathematics with determination. Last but not least, I would like to thank my parents, and it's they who are always supporting me behind.

\section{CONFLICTS OF INTEREST}

The author declares no conflicts of interest regarding the publication of this paper.

\section{REFERENCES}

1. Jordan, C. (1887) Cours d'Analyse de l' Ecole Polytechnique. Gauthier-Villars, 3, 587-594.

2. Veblen, O. (1905) Theory on Plane Curves in Non-Metrical Analysis Situs. Transactions of the American Mathematical Society, 6, 83-98. https://doi.org/10.2307/1986378

3. Hales, T. (2007) Jordan's Proof of the Jordan Curve Theorem. Studies in Logic, Grammar and Rhetoric, 10, 45-60.

4. Schoenflies, A. (1924) Bemerkungen zu den Beweisen von C. Jordan und Ch. J. de la Vallée Poussin, Jahresber. Deutsche Mathematiker-Vereinigung (DMV), 33, 157-160.

5. Filippov, A.F. (1950) An Elementary Proof of Jordan's Theorem. Uspekhi Mat. Nauk, 5, 173-176. (In Russian)

6. Tverberg, H. (1980) A Proof of the Jordan Curve Theorem. Bulletin of the London Mathematical Society, 12, 34-38. https://doi.org/10.1112/blms/12.1.34

7. Maehara, R. (1984) The Jordan Curve Theorem via the Brouwer Fixed Point Theorem. The American Mathematical Monthly, 91, 641-643. https://doi.org/10.1080/00029890.1984.11971517

8. Thomassen, C. (1992) The Jordan-Schönflies Theorem and the Classification of Surfaces. American Mathematical Monthly, 99, 116-130. https://doi.org/10.1080/00029890.1992.11995820

9. Pugh, C.C. (2016) Real Mathematical Analysis. Springer, New York, 129. 